\title{
Space Awareness: Inspiring A New Generation of Space Explorers
}

\author{
Pedro Russo ${ }^{1}$ \\ (on behalf of the Space Awareness Project Consortium) \\ ${ }^{1}$ Leiden Observatory / Leiden University, the Netherlands \\ email:russo@strw.leidenuniv.nl
}

\section{Overview}

Space Awareness (EUSPACE-AWE) will use the excitement of space to attract young people into science and technology and stimulate European and global citizenship. Our main goal is to increase the number of young people that choose space-related careers.

EUSPACE-AWE targets diverse groups that are influential in the complex processes that lead to career decisions. The project will show teenagers the opportunities offered by space science and engineering and inspire primary-school children when their curiosity is high, their value systems are being formed and seeds of future aspirations are being sown.

A carefully crafted portfolio of EUSPACE-AWE activities will: 1. Acquaint young people with topical cutting-edge research and "role-model" engineers, 2. Demonstrate to teachers the power of space as a motivational tool and the opportunities offered by space careers, 3. Provide a repository of innovative peer-reviewed educational resources, including toolkits highlighting seductive aspects of Galileo and Copernicus and 4. Set up a "space career hub" and challenging contest that will appeal to teenagers. Attention will be paid to stimulating interest amongst girls and ethnic minorities and reaching children in underprivileged communities, where most talent is wasted. Targeting policy makers via high-impact events will help ensure sustainability and demonstrate the social value of the space programme.

EUSPACE-AWE shall maximise cost effectiveness of the activities by joining with and supplementing existing space teacher training networks and courses and exploiting and expanding infrastructures of the proven FP7-Space projects, EU Universe Awareness for young children and Odysseus for teenagers.

EUSPACE-AWE will join with and complement existing space-education programs and be coordinated closely with the European Space Agency (ESA). We shall reach European teachers, schools and national curricula through the national host organisations of ESA's European Space Education Resource Offices (ESEROs) and the extensive networks of European Schoolnet, (Scientix) and Universe Awareness (UNAWE). Designated EUSPACE-AWE nodes will provide curriculum and resource localisation and test beds for academic evaluation. A partnership with the IAU Office of Astronomy for Development in Cape Town (South Africa) will ensure global reach.

More information: www.space-awareness.org 